\title{
3D Sensors on Driverless Trucks for Detection of Overhanging Objects in the Pathway
}

\section{Citation}

Hedenberg, K. and Åstrand, B., "3D Sensors on Driverless Trucks for Detection of Overhanging Objects in the Pathway," Autonomous Industrial Vehicles: From the Laboratory to the Factory Floor, ASTM STP1594, R. Bostelman and E. Messina, Eds., ASTM International, West Conshohocken, PA, 2016, pp. 41-56, doi:10.1520/STP159420150051

\begin{abstract}
Human-operated and driverless trucks often collaborate in a mixed work space in industries and warehouses. This is more efficient and flexible than using only one kind of truck. However, because driverless trucks need to give way to driven trucks, a reliable detection system is required. Several challenges exist in the development of such a system. The first is to select interesting situations and objects. Overhanging objects are often found in industrial environments (e.g., tines on a forklift). Second is choosing a system that has the ability to detect those situations. (The traditional laser scanner situated two decimetres above the floor does not detect overhanging objects.) Third is to ensure that the perception system is reliable. A solution used on trucks today is to mount a two-dimensional laser scanner on top and tilt the scanner toward the floor. However, objects at the top of the truck will be detected too late, and a collision cannot always be avoided. Our aim is to replace the upper two-dimensional laser scanner with a three-dimensional camera, structural light, or time-of-flight (TOF) camera. It is important to maximize the field of view in the desired detection volume. Hence, the sensor placement is important. We conducted laboratory experiments to check and compare the various sensors' capabilities for different colors, using tines and a model of a tine in a controlled industrial environment. We also conducted field experiments in a warehouse. Our conclusion is that both the
\end{abstract}

\footnotetext{
Manuscript received June 15, 2015; accepted for publication November 3, 2015.

'University of Skövde, School of Engineering Science, Portalen, Kaplansgatan 11, SE-541 34 Skövde, Sweden

${ }^{2}$ Halmstad University, School of Information Technology, Kristian IV:S väg 3, SE-30118 Halmstad, Sweden

${ }^{3}$ ASTM Workshop on Autonomous Industrial Vehicles: From the Laboratory to the Factory Floor on

May 26-30, 2015 in Seattle, Washington.
} 
tested structural light and TOF sensors have problems detecting black items that are non-perpendicular to the sensor. It is important to optimize the light economy-meaning the illumination power, field of view, and exposure time-in order to detect as many different objects as possible.

\section{Keywords}

mobile robots, safety, obstacle detection

\section{Introduction}

The need for an obstacle detection system for driverless forklift trucks is obvious. However, such a system also may be used on driven trucks with automated functions, such as the ability to stop if an obstacle appears. Hence, developing systems that can be implemented for driven and driverless forklift trucks will not only create a safer environment but will also decrease the cost of the obstacle detection system. The world market for driven forklift trucks is orders of magnitude higher than for driverless forklift trucks. American companies sold 927 automated guided vehicles (AGVs) in 2011 [1]. During that period, more than 200,000 forklift trucks were sold in the United States [2]. This includes electric rider trucks, electric warehouse rider trucks, electric warehouse pedestrian trucks, and internal combustion trucks. For the world market, World Industrial Truck Statistics reported order bookings slightly below one million driven trucks for 2011 [2].

Two different safety standards exist for driverless trucks, one for Europe (EN1525) and one for the United States (ANSI/ITSDF B56.5-2012), and each has developed differently. In terms of obstacles, both consider contact with humans and have two test items that represent parts of a human-a lower leg and a body. However, an object representing a piece of a machinery is added to the U.S. standard, and the standard also considers different materials for different sensors as well as more test cases $[3,4]$. A continuous development of standards for driverless trucks is important to make use of state-of-the-art sensor technology.

Overall, the motivation for this work is to improve the safety of automated material handling by proposing better sensor solutions for obstacle detection. The challenge in developing an obstacle detection system in industrial settings is threefold. The first is to select situations to detect that are of special interest. The second is choosing a perception system that has the ability to detect those situations. The third is to ensure that the perception system is reliable.

This chapter is organized as follows. First, we discuss related work. This is followed by a problem definition and then descriptions of the experiments and the results. Finally, we offer a discussion and conclusions drawn from the results.

\section{Related Work}

The National Institute of Standards and Technology has presented covering standards for driverless forklift trucks in industrial environments. Bostelman, 
Hong, and Madhavan [5] use a time-of-flight (TOF) camera to detect objects described in the U.S. safety standard [4] in which they also test the camera outdoors and conclude that it shows good results in shady environments. Bostelman [6] conducts tests with a sonar, a two-dimensional lidar, and a three-dimensional TOF camera. The sensors are tested against objects in the standards as well as an additional item, $500 \mathrm{~mm}$ by $100 \mathrm{~mm}$, posed at $0^{\circ}$ and $45^{\circ}$ to the robot's direction of travel to make a difference for TOF sensors. The tests also included various materials (e.g., cardboard, gray plastic, cotton denim, black reflectance paper, aluminum, and clear glass). Sonar detected all objects in various materials but had problems with different angles. The two-dimensional lidar had problems with flat glass at a $45^{\circ}$ angle but detected other objects. The three-dimensional TOF camera showed a notable difference between highly reflective and low-reflective materials. Bostelman [6] proposed changes to ANSI/ITSDF B56.5 that were later adopted into the standard [4]. Bostelman, Norcross, Falco, and Marvel proposed test methods for driverless trucks and give examples of potential human and equipment effects on the path of a driverless truck in human/AGV collaborative work spaces [7].

Hedenberg and Åstrand use a test apparatus (Fig. 1) to evaluate three-dimensional sensors that include test items in the safety standard as well as new items that represent objects in an industrial environment [8].

\section{Problem Definition}

\section{SENSORS FOR OBSTACLE DETECTION}

A driverless truck equipped with a two-dimensional range scanner (e.g., laser scanner) situated less than two decimetres above the floor to detect objects described in the safety standards (ANSI/ITSDF B56.5-2012, EN1525) does not detect all obstacles in the desirable detection volume-the yellow area in Fig. 2. A solution used on trucks today is to mount a two-dimensional laser scanner on the top of the truck and tilt the scanner toward the floor. However, objects on the top of the truck will be detected too late, and a collision cannot always be avoided. Another solution proposed by Bostelman, Shackleford, Cheok, and Saidi is to use laser scanners on each side of the truck to detect all items that enter the contour area of the truck [9]. However, this will dramatically increase sensor costs.

Our aim is to replace the upper two-dimensional laser scanner with a threedimensional camera in order to increase the detection volume and thus detect obstacles earlier compared to the systems used today (Fig. 2).

For all vision systems, the placement of the cameras is essential for obtaining a good result. This has to be considered for every new setup [10]. There are several ways to determine the placement of the camera system on the robot. The easiest way is to just choose a placement by intuition. Putting a little more effort into this judgment will probably increase the precision in the system. It will also make a discussion of camera placement more unbiased if the system's 
FIG. 1 The test apparatus. In one scene, the test rig represents a collection of objects: Item A-a prone human, Item B-a standing human, Item C-a flat target used by Bostelman and Shackleford [6], Item D-a ladder, Item E-tines on a forklift, Item F-hanging cable, Items $\mathrm{G}$ and $\mathrm{H}$-vertical bars, Item I-horizontal bar, and Item J-thin cable. A ladder, Item D, typically has a steeper slope than $45^{\circ}$. However, objects that have a larger angle may be considered vertical, while objects with a lower angle can be considered horizontal. The test apparatus measures $1.8 \mathrm{~m}$ by $1.8 \mathrm{~m}$ and the bars have a thickness of $25 \mathrm{~mm}$. The hanging cable has a diameter of $13 \mathrm{~mm}$. All items are painted in matt black.

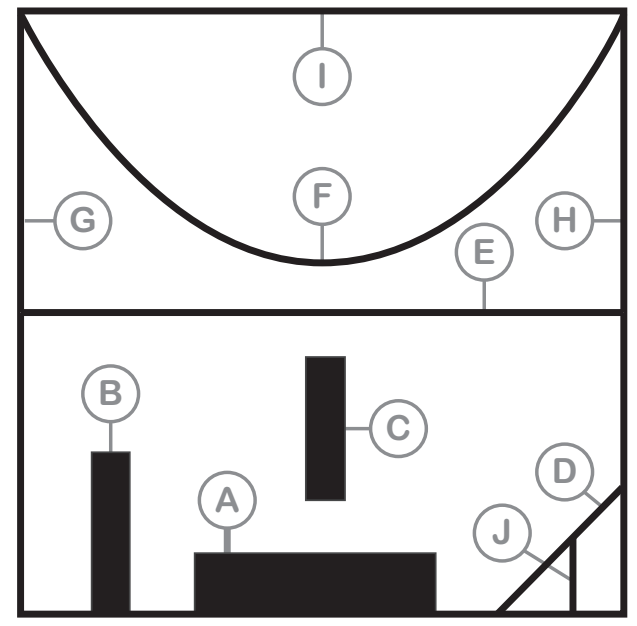

performance has to be increased later. Huang and Krotkov concluded that the best placement for cameras on mobile robots is at the highest point possible [10]. This is true if the desired detection volume is small in comparison to the available field of view (FOV). In other cases, maximizing the FOV in the desired detection volume is necessary.

\section{OBJECTS IN THE ENVIRONMENT}

Obstacles used in academic studies about mobile robots in indoor environments are often box- or cylindrical-shaped (e.g., cans) or are humans. However, a closer look at the industry will identify other obstacles. Detecting these obstacles can be as important to human safety as direct detection of humans. Four examples of typical situations found in an industry environment are shown in Fig. 3 and include a protruding bar, hanging tools, blocking tape, and a ladder. None of these shows a typical box- or cylindrical-shaped form. A common denominator for these objects is their relatively thin structure and the fact that they overhang.

Forklift tines are other common objects in industrial environments and need to be considered because both human-operated and driverless trucks collaborate in 
FIG. 2 Configuration of the sensor system on a driverless truck. A two-dimensional laser scanner mounted to detect obstacles below $2 \mathrm{dm}$ above the floor is used to meet the safety standards. Overhanging objects can be detected by a tilted two-dimensional laser scanner mounted on the top of the truck. This solution will not detect all obstacles in the desired detection volume (yellow area). A three-dimensional camera covers a larger volume.

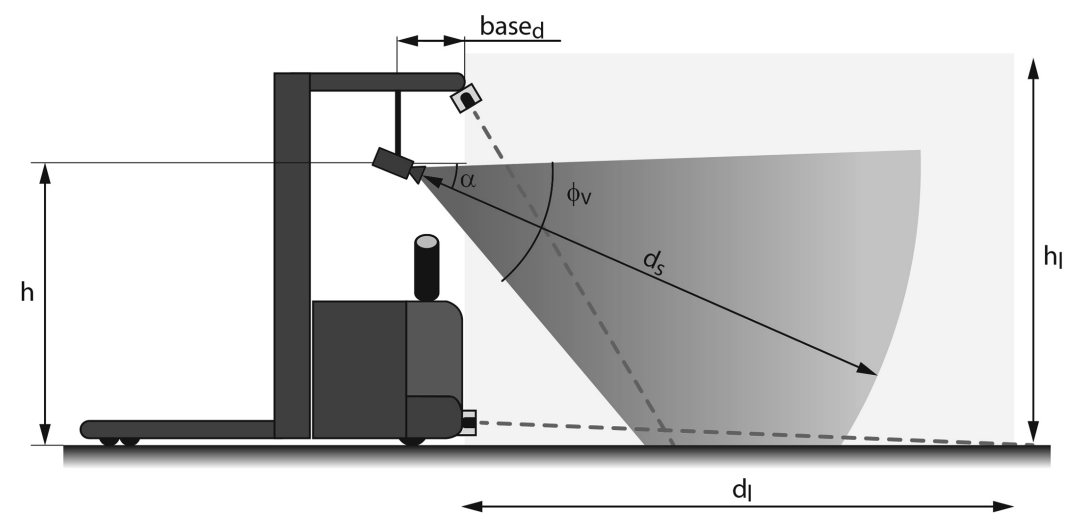

the same work space, Fig. 4. On a system level, it is no problem to keep track of the position and the action taken for all the driverless trucks, but a similar system for human-operated trucks is more problematic. A driverless truck must give way to a human-operated truck, and the lower two-dimensional laser scanner on a driverless truck can indicate the presence of a human-operated truck. One problem with this is if the human-operated truck delivers goods in front of a driverless truck (Fig. 4). The laser scanner will not detect the tines, presenting the risk of the driverless truck running into the human-operated truck.

The problem with the coverage of the desired detection volume and FOV of a structural light sensor is demonstrated in Fig. 4. The FOV hardly covers the floor close enough to the truck and at the top of the truck's contour area.

Other similar scenarios are turns around corners (Fig. 5) as described earlier [7]. Mirrors are often used in human work spaces to see around corners. For driverless trucks, this is a more complex scenario and still is an open issue.

To illustrate the complexity in an industrial environment, Fig. 4b shows how spilled water may change the environment for sensor systems. The wet spot on the otherwise matt floor may cause objects to be reflected, which reduces the optical signal returning to the sensor. This could cause false readings.

In the existing safety standards for driverless trucks, objects commonly handled are those that represent humans $[3,4]$. Since 2012, the U.S. standard also has a $500 \mathrm{~mm}$ by $500 \mathrm{~mm}$ plate that needs to be detected at $0^{\circ}$ and $45^{\circ}$ with different colors and reflectance depending on the sensor used. 
FIG. 3 Objects relevant for obstacle detection in an industrial environment. A common denominator for these objects is that they are thin and mostly overhanging. Objects on images (a)-(c) are also overhanging. Images (a)-(c) are published with permission from Volvo Group Trucks Operations; $(d)$ is published with permission from Volvo Car Corporation.

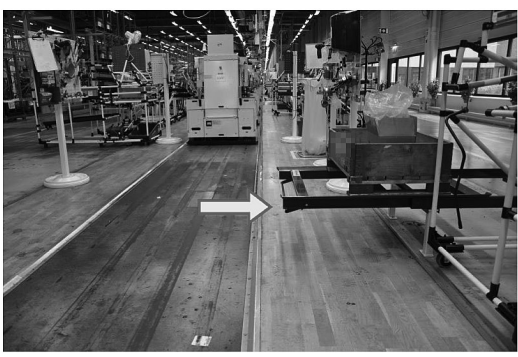

(a)

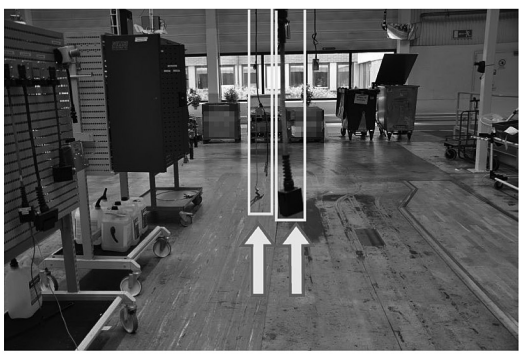

(c)

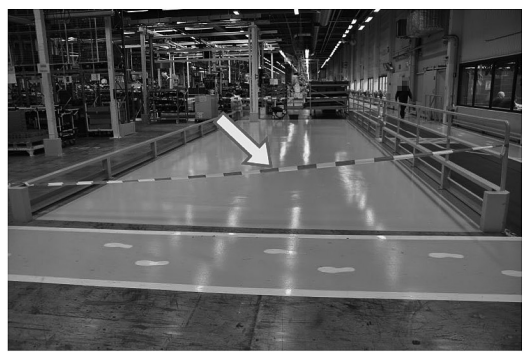

(b)

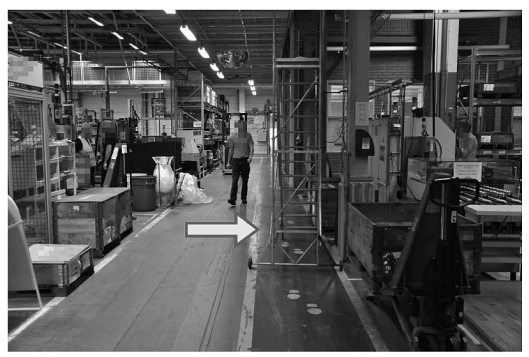

(d)

\section{Experiments and Results}

\section{TEST APPROACH}

Our aim is to replace the upper two-dimensional laser scanner with a threedimensional camera and to make early calculations on how to maximize the FOV in the desired detection volume. For many reasons, a high position seems advantageous. However, early tests with different sensor positions also showed that reflectance on untextured regions may have a high impact on the sensor readings. Therefore, this caused some initial tests to identify the importance of the color of the test items and their effect on sensor placement.

We also conducted experiments with different sensors in a controlled industrial environment and made real-field experiments in a warehouse using a combination of driverless and human-operated trucks.

\section{TIME-OF-FLIGHT AND STRUCTURAL LIGHT SENSORS ON UNTEXTURED REGIONS WITH DIFFERENT COLORS}

An experiment was conducted to investigate whether there are any performance differences in TOF and structured light cameras on untextured regions with different colors. 
FIG. 4 View from a driverless truck. Human-operated and driverless trucks cooperate in many industrial environments. A human-operated truck delivers goods in front of a driverless truck. The two-dimensional laser scanner on the driverless truck indicates the presence of the human-operated truck and stops. Note the wet spot on the otherwise matt floor behind the truck, which is more visible in (b). This makes a reflective surface that might cause problems for some sensors.

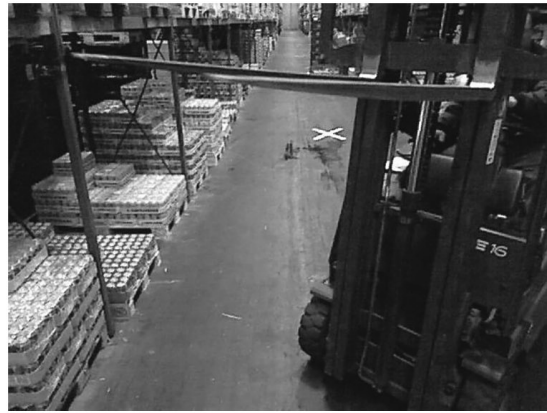

(a)

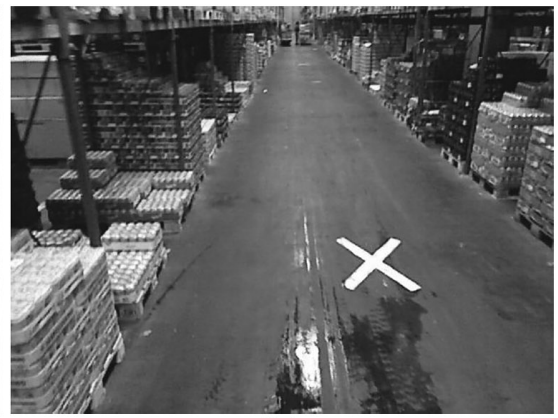

(b)

FIG. 5 An AGV with a bumper turns around a pillar. Note the window to the left in the left image. Sunlight may have an impact on three-dimensional sensors. Published with permission from Volvo Car Corporation.

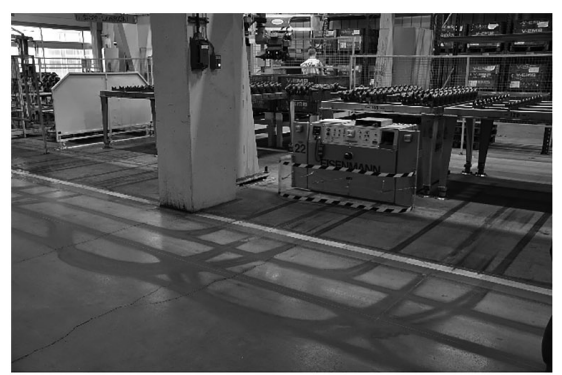

(a)

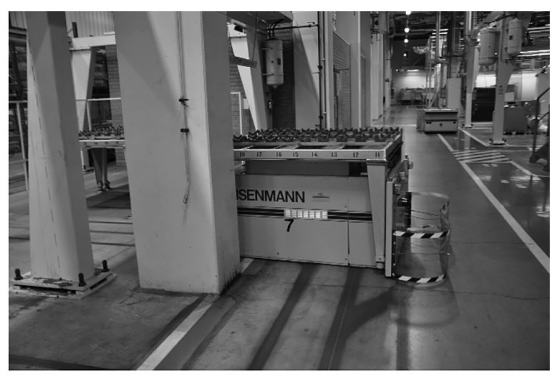

(b)

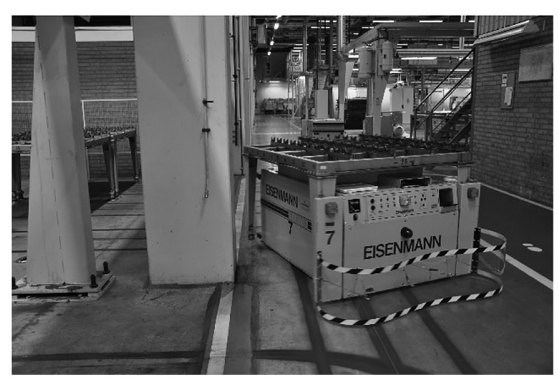

(c) 
TABLE 1 Results for the structured light camera. The camera has problems with black colors at almost all angles. The results are rounded off to the closest $10 \%$ value. The results are not symmetrical due to different distances to the board at different angles.

\begin{tabular}{l|c|c|c|c}
\multicolumn{5}{c}{ Structured light camera } \\
\hline Angle & Gray & White & Red & Black \\
\hline$-45^{\circ}$ & $100 \%$ & $100 \%$ & $100 \%$ & $0 \%$ \\
$-15^{\circ}$ & $100 \%$ & $100 \%$ & $100 \%$ & $100 \%$ \\
$0^{\circ}$ & $100 \%$ & $100 \%$ & $100 \%$ & $100 \%$ \\
$+15^{\circ}$ & $100 \%$ & $100 \%$ & $100 \%$ & $0 \%$ \\
$+45^{\circ}$ & $100 \%$ & $100 \%$ & $100 \%$ & $0 \%$ \\
\hline
\end{tabular}

A TOF camera (Fotonic E70) and a structured light camera (Microsoft Kinect) were used and placed $2.5 \mathrm{~m}$ from a piece of board painted in four different colors: gray, white, red, and black. A white/neutral background was placed $1 \mathrm{~m}$ behind the board. The board was arranged at five different angles: $-45^{\circ},-15^{\circ}, 0^{\circ},+15^{\circ}$, and $+45^{\circ}$. Obstacles at an angle of $-45^{\circ}$ are used in the U.S. safety standard [4]. Three consecutive images were taken by each sensor. The analysis was made by counting the number of pixels within a given depth value from the sensor. Then the ratio between the number of detected pixels and the number of maximum possible pixels covered by the sensor was computed. The average of the results from the three images is given in Table 1 and Table 2, and a scene is shown in Fig. 6. The results were rounded off to the closest $10 \%$ value.

It is clear that TOF and structured light cameras perform well for untextured regions. However, this depends heavily on the reflectance. Both sensors performed poorly on black surfaces in this test due to the fact that the black color had poor reflectance. Usually, black is a good light absorber, but reflectance is important, and one issue is to define the reflectance for different wavelengths. The structured light sensor performed better on the perpendicular flat object in black. The board was

TABLE 2 Results for the time-of-flight camera. The camera has problems with black colors at almost all angles and also with the gray plate at $-45^{\circ}$. The results are rounded off to the closest $10 \%$ value.

TOF camera

\begin{tabular}{l|c|c|c|c}
\hline Angle & Gray & White & Red & Black \\
\hline$-45^{\circ}$ & $60 \%$ & $100 \%$ & $100 \%$ & $0 \%$ \\
$-15^{\circ}$ & $100 \%$ & $100 \%$ & $100 \%$ & $0 \%$ \\
\hline $0^{\circ}$ & $100 \%$ & $100 \%$ & $100 \%$ & $60 \%$ \\
$+15^{\circ}$ & $100 \%$ & $100 \%$ & $100 \%$ & $0 \%$ \\
$+45^{\circ}$ & $100 \%$ & $100 \%$ & $100 \%$ & $0 \%$ \\
\hline
\end{tabular}


FIG. 6 The plate at $+15^{\circ}$ rotated about the vertical axis. The time-of-flight (b) and structured light (c) camera do not detect the black plate. All other colors are detected by both sensors.

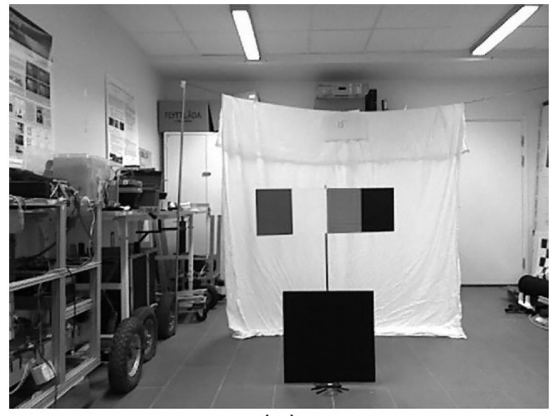

(a)

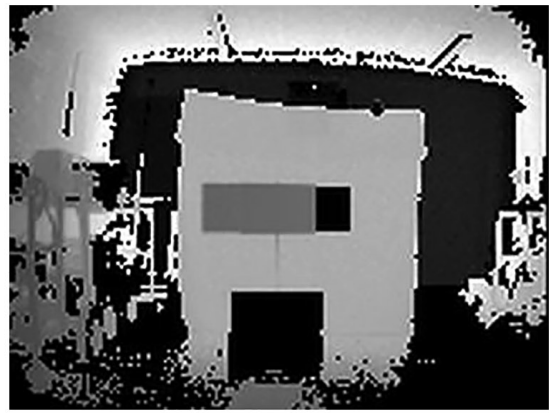

(b)

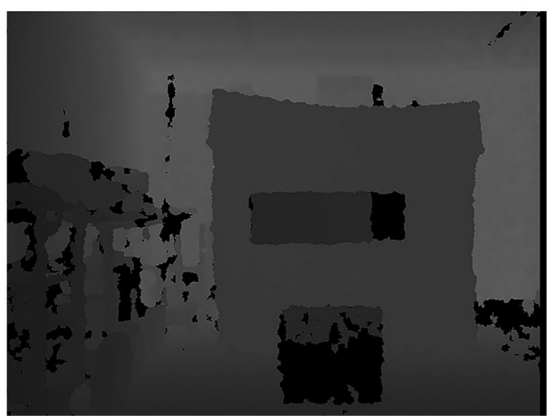

(c)

FIG. 7 A time-of-flight and structural light camera were mounted on a driverless truck. A scenario is to turn around a corner and detect tines on a forklift.

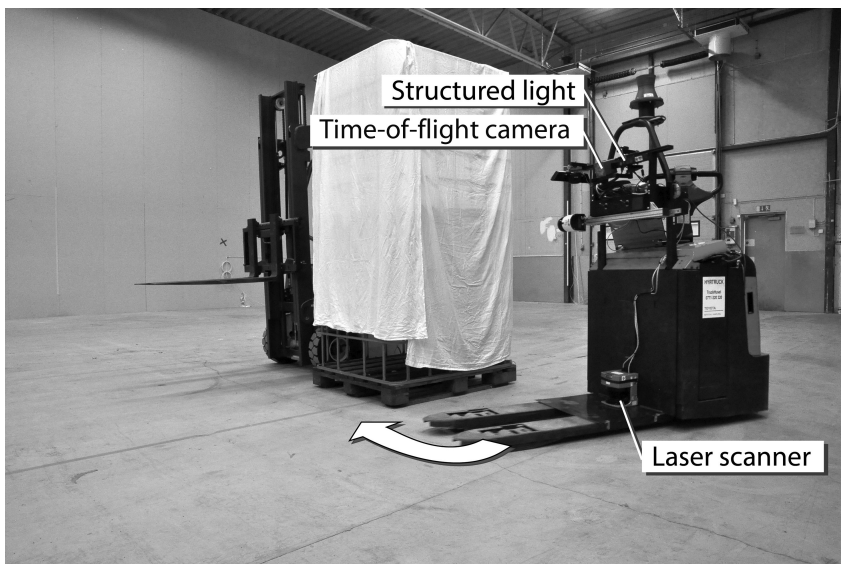


rather diffuse, meaning that the light was scattered in all directions. If the board had been more specular, we would probably have seen an even larger effect by tilting it. The results in Table 1 are not symmetric due to the different distances to the board at different angles.

\section{TIME-OF-FLIGHT AND STRUCTURAL LIGHT SENSORS IN A CONTROLLED INDUSTRIAL ENVIRONMENT}

To investigate the performance of TOF and structural light sensors in detecting tines, experiments were conducted in an industrial environment. A TOF camera (Fotonic E70) and a structured light camera (Microsoft Kinect) were mounted on a driverless truck and programmed to turn around a corner (Fig. 7). A laser scanner (lidar) served as a reference. Two different objects were used: the tines of a forklift

FIG. 8 The results from a scenario where tines from a forklift are used as an obstacle around the corner. In the upper left, an RGB image from the Kinect is shown. The lower left shows the two-dimensional plot from the laser scanner in which the red line illustrates where the beam from the upper laser scanner hits the floor. The upper right image shows the depth image from the structured light (Kinect) camera. The lower right image shows the depth image from the TOF camera (Fotonic). The structured light camera (Kinect) as well as the TOF camera (Fotonic) detect the tines. Note the problems for the TOF camera with the reflectors mounted on the wall and used for laser navigation.
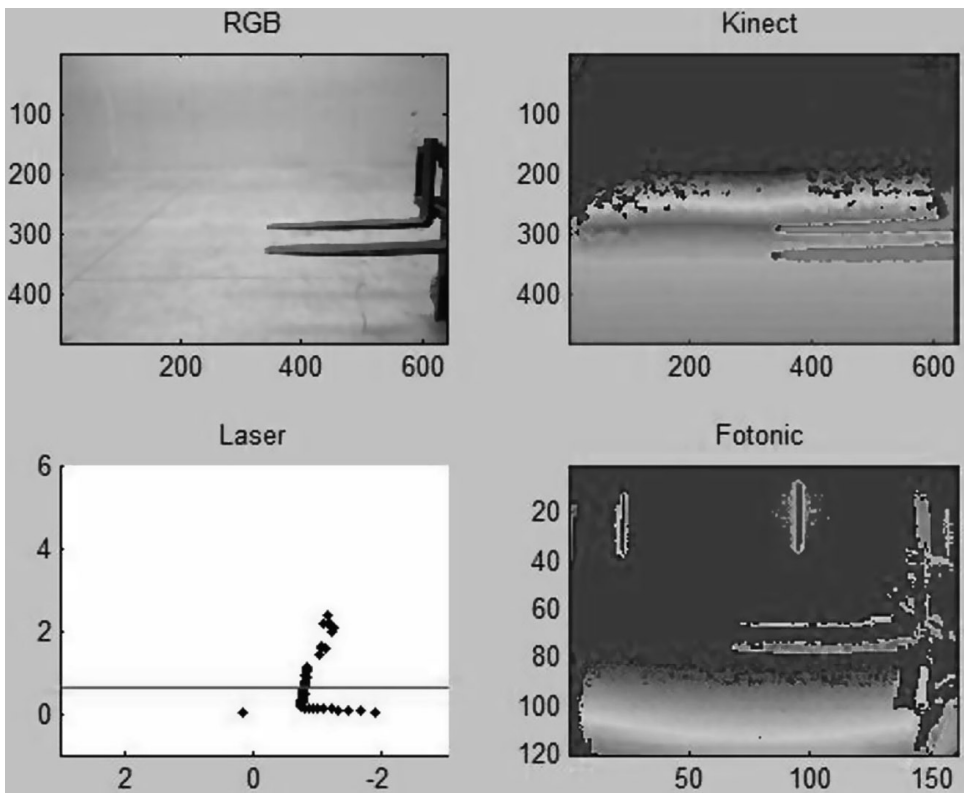
and a model of a tine. The approach in the safety standards is to make a model of an existing object and conduct tests. If the model is detected, the perception system is approved for that specific situation $[3,4]$. The model was painted black where the tine of the forklift had been worn off naturally. We make no claim that either the model or the real tine represent the average tines used in the industry. Our investigation showed that it is very hard to find a common denominator for making one general model of a tine.

The results are shown in Fig. 8 and Fig. 9. The lower left image shows the two-dimensional plot from the laser scanner in which the red line illustrates where the beam from the upper laser scanner hits the floor. The upper right image shows the depth image from the structured light (Kinect) camera. The lower right image shows the depth image from the TOF camera (Fotonic). Both the structured light camera (Kinect) and the TOF camera (Fotonic) detected the

FIG. 9 The results from a scenario where a model represents a tine from a forklift and is used as an obstacle around a corner. On the upper left, an RGB image from the Kinect is shown. The lower left image shows the two-dimensional plot from the laser scanner in which the red line illustrates where the beam from the upper laser scanner hits the floor. The upper right image shows the depth image from the structured light (Kinect) camera. The lower right image shows the depth image from the TOF camera (Fotonic). The structured light camera (Kinect) detects the model of the tine, while the TOF camera (Fotonic) has difficulty detecting the model.
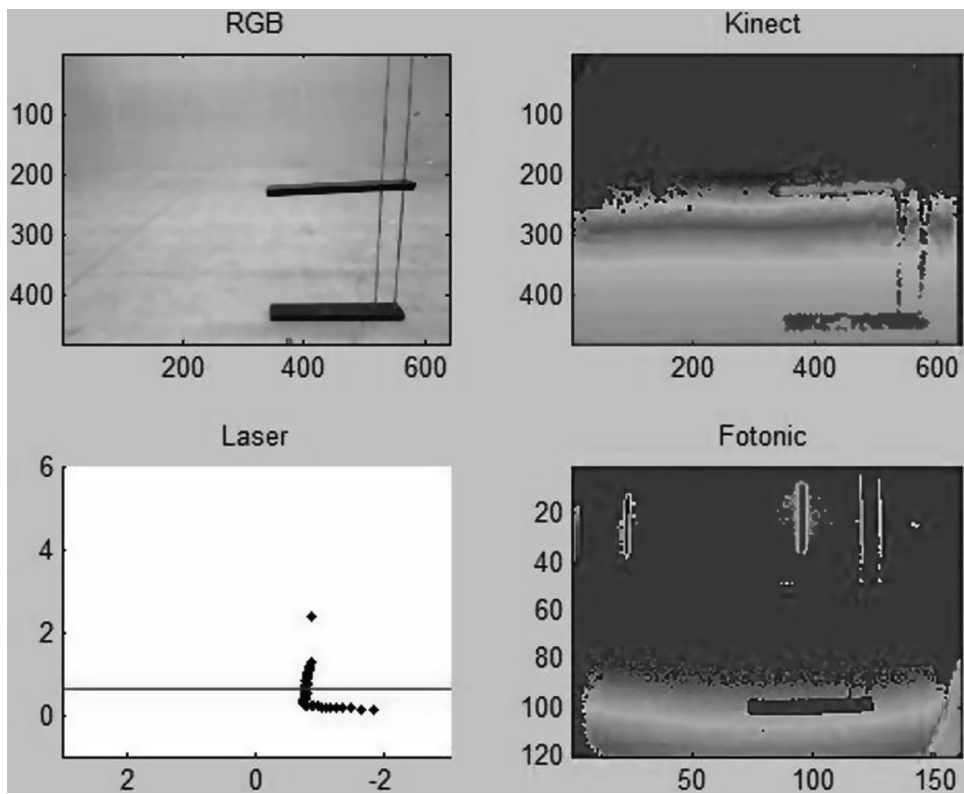
model of the tine. It is also notable that the TOF camera had problems with the reflectors used for navigation (see Fig. 8). The test items in the U.S. safety standard were also used. Both sensors had difficulty detecting the black flat test item but did detect the cylindrical black test pieces.

It is clear that the structured light sensor detects both objects, whereas the TOF sensor has problems with the model of the tine. According to the test mentioned earlier, this is due to the black color of the model; but another reason can be a lower resolution ( 160 by 120 versus 320 by 240 ) with a higher FOV $\left(70^{\circ}\right.$ by $53^{\circ}$ versus $58.5^{\circ}$ by $46.6^{\circ}$ ) for the TOF sensor compared to the structural light sensor. There are not enough pixels to detect objects of this size.

\section{FIELD TEST IN A WAREHOUSE}

We made a field test in a warehouse where driverless and human-operated trucks collaborate in the same work space. A structural light camera (Kinect) was mounted on a driverless truck close to the upper laser scanner. The truck was programmed

FIG. 10 Field test from a warehouse where a structured light camera is used A wooden broomstick is placed in front of the truck. The thin stick is detected by the sensor. The dark blue color in the depth image indicates undefined distances. The lower left image shows the two-dimensional plot from the laser scanner in which the red line illustrates where the beam from the upper laser scanner hits the floor. Detections of the truck are shown at $(\mathrm{O}, \mathrm{O})$.
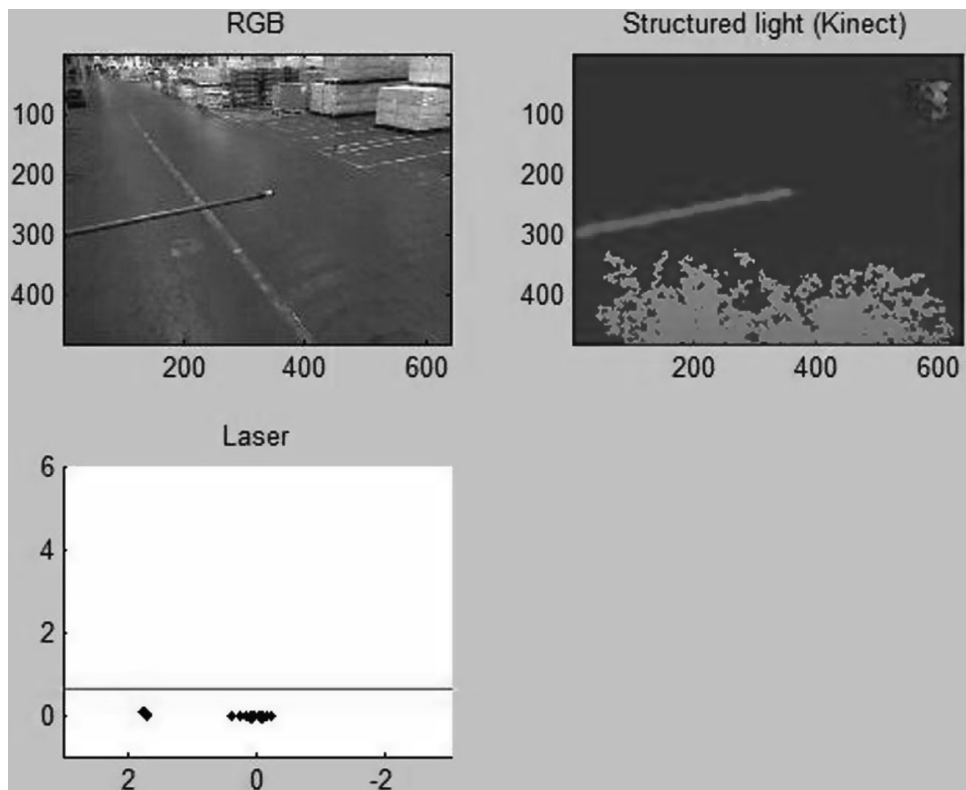
to make several laps throughout the warehouse. Humans and driverless and human-operated trucks occurred as obstacles as well as manually placed obstacles to test the system's limitations; see Figs. 10-12.

The sensor detected thin structures, such as the broomstick in Fig. 10 and the metal cage in Fig. 12, but had problems with the black parts in the black-yellow pattern of the safety railing in Fig. 11.

\section{Discussion and Conclusion}

We conducted tests to determine if active three-dimensional sensors can be used for obstacle detection in industrial environments and for replacing an upper twodimensional laser scanner on a driverless truck.

The initial tests identified the influence different colors have on flat untextured regions for active three-dimensional range sensors. The results showed that a black color with low reflectance on test items used in safety standards and in our earlier

FIG. 11 Field test from a warehouse where a structured light camera is used. The bar in black and yellow (on the left) is only partly detected by the sensor. The black part is not detected. The dark blue color in the depth image indicates undefined distances. The lower left image shows the two-dimensional plot from the laser scanner in which the red line illustrates where the beam from the upper laser scanner hits the floor. Detections of the truck are shown at $(0,0)$.
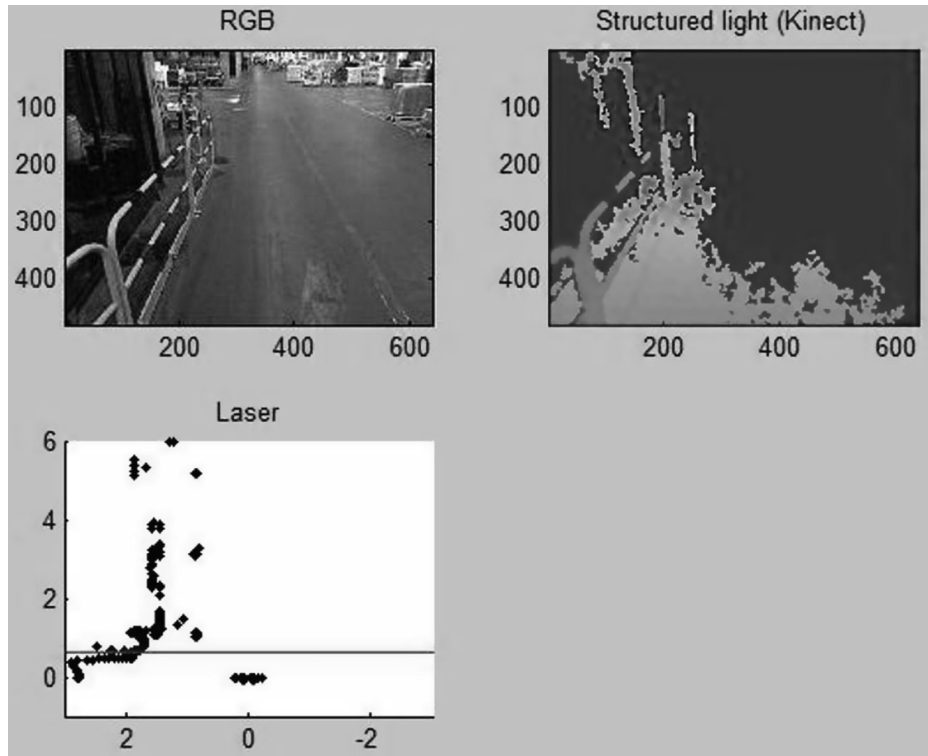
proposed test apparatus is relevant. Black plates at nonperpendicular angles only return a low signal to the sensors, and these objects were difficult for the tested TOF and structural light sensors to detect. This has an impact on the camera placement.

The maximum detection range is shorter for low-reflective objects, implying that the geometry and camera should be optimized for the darkest object that should be detectable and at the minimum critical distance. The dynamic range of the sensors is also very important in order to handle different colors at varying distances. Multi-shutter modes to increase the dynamic range would also be interesting to evaluate. The difficulty for structural light cameras to detect black items was verified in the field experiments. The TOF camera has problems with the reflectors for navigation as well as with the black areas on the test items. This may make the TOF camera more problematic on driverless trucks where laser navigation with reflectors is used.

We also compared different results from TOF and structural light cameras in a controlled industrial environment. Fork tines on a forklift and a model of a forklift

FIG. 12 Field test from a warehouse where a structured light camera is used. A metal cage is placed in front of the truck. The thin structures are detected by the sensor. The dark blue color in the depth image indicates undefined distances. The lower left image shows the two-dimensional plot from the laser scanner in which the red line illustrates where the beam from the upper laser scanner hits the floor. Detections of the truck are shown at $(0,0)$.
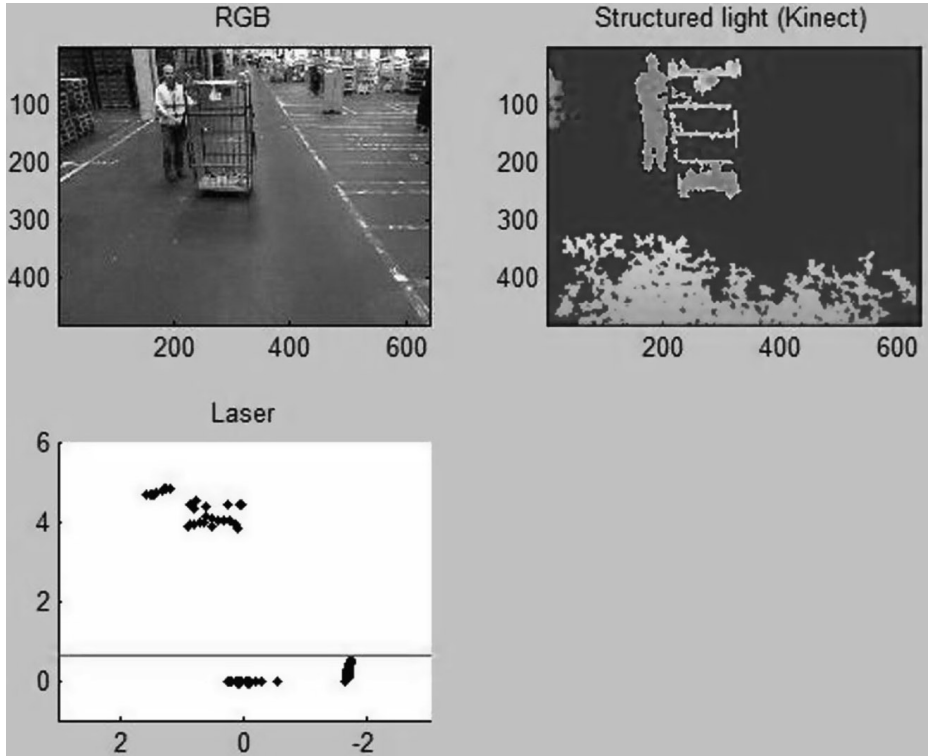
tine were used as obstacles. Both sensors detected the tine. However, the TOF camera had problems detecting the black tine model, while the structural light camera performed better. The results show that a black color with low reflectance is hard for TOF and structural light cameras to detect. They also show how difficult it is to make a representative model of tines for a forklift. To our knowledge, making a tine model (or a representative test of a tine model) to be used in a safety standard is still an open issue.

To determine a good placement for a three-dimensional sensor, a large FOV is necessary to cover the desired detection volume. The placement of the sensor is more important due to the poor sensor performance in detecting black plates at nonperpendicular angles. Active three-dimensional sensors currently on the market have a limited FOV. Sensors with a larger FOV are more suitable for this task, without losing resolution to detect thin structures. This may require infrared light with more power. It may also require different structural light patterns more suitable for detecting obstacles in an industrial environment.

\section{ACKNOWLEDGMENTS}

As a part of the Automatic Inventory and Mapping of Stock project, this work is supported by the Swedish Knowledge Foundation and by industry partners Kollmorgen, Optronic, and Toyota Material Handling Europe.

\section{References}

[1] Material Handling Institute, "AGVS Quarterly Report,” MHI, Charlotte, NC, Summer 2012.

[2] European Federation of Materials Handling, "World Industrial Truck Statistics," Information Sheet, July 2012, Frankfurt/Main, http://www.fem-eur.com/data/File/N460-WITS_ fact_sheet_2012_FEM_corr2.pdf (accessed April 4, 2016).

[3] European Committee for Standardization (CEN), "Safety of Industrial Trucks-Driverless Trucks and Their Systems,” CEN, Brussels, Belgium, 1997.

[4] ANSI/ITSDF B56.5, "Safety Standard for Driverless, Automatic Guided Industrial Vehicles and Automated Functions of Manned Industrial Vehicles," Industrial Truck Standards Development Foundation, Washington, DC, 2012.

[5] Bostelman, R., Hong, T., and Madhavan, R., "Towards AGV Safety and Navigation Advancement Obstacle Detection Using a TOF Range Camera," Proceedings of the 12th International Conference on Advanced Robotics, Seattle, WA, July 18-20, 2005, Institute of Electrical and Electronics Engineers (IEEE), New York, 2005, pp. 460-467.

[6] Bostelman, W. and Shackleford, R., "Time of Flight Sensors Experiments Towards Vehicle Safety Standard Advancements," Draft submitted to the Computer Vision and Image Understanding special issue on Time of Flight Sensors, 2010.

[7] Bostelman, R., Norcross, R., Falco, J., and Marvel, J., "Development of Standard Test Methods for Unmanned and Manned Industrial Vehicles Used Near Humans," Proceedings of the SPIE Defense, Security, and Sensing Conference, Baltimore, MD, April 
29-May 3, 2013, National Institute of Standards and Technology (NIST), Gaithersburg, MD, 2013.

[8] Hedenberg, K. and Åstrand, B., "Safety Standard for Mobile Robots-A Proposal for 3D Sensors," Proceedings of the 5th European Conference on Mobile Robots, Örebro, Sweden, September 7-9, 2011, Centre for Applied Autonomous Sensor Systems, Örebro University, Örebro, Sweden, 2011, pp. 235-252.

[9] Bostelman, R., Shackleford, W., Cheok, G., and Saidi, K., "Safe Control of Manufacturing Vehicles Research Towards Standard Test Methods," Proceedings of the International Material Handling Research Colloquium 2012 (IMHRC 2012), Gardanne, France, June 25-28, 2012, NIST, Gaithersburg, MD, 2012.

[10] Huang, W. H. and Krotkov, E. P., "Optimal Stereo Mast Configuration for Mobile Robots," in Proceedings of the IEEE International Conference on Robotics and Automation, Vol. 3, Albuquerque, NM, April 20-25, 1997, IEEE, New York, 1997, pp. 1946-1951. 